\title{
Prospección de la estimación de la evapotranspiración de referencia, bajo las condiciones del valle de Chaca, Arica-Chile
}

\author{
Research of the estimation of the evapotranspiration of reference, \\ under the conditions of Chaca's valley, Arica-Chile
}

Amador Torres Hernández ${ }^{1 *}$, Rodrigo Vásquez Vásquez

\begin{abstract}
RESUMEN
A partir de los datos meteorológicos obtenidos en la estación meteorológica automática (EMA) -instalada en el valle de Chaca de la Región de Arica y Parinacota, Chile- se comparó la ETo estimada con el método de Blaney Criddle, Radiación, Penman 1948, Hargreaves Samani 1985 y Bandeja de Evaporación con la estimada mediante el método de Penman Monteith FAO 56. No se obtuvo resultado satisfactorio con la ecuación Blaney Criddle (RMSE 6,35 mm/día y $\mathrm{R}^{2}$ 67\%). El método de radiación subestimó la Eto, con valores similares a los obtenidos con Penman 1948, en tanto la bandeja evaporación sobrestima considerablemente a medida que suben las temperaturas. Finalmente, el método de Hargreaves Samani 1985 presentó el mejor ajuste con los valores de ETo estimados con Penman Monteith FAO 56, utilizando solo la temperatura del aire y radiación solar, lo cual permite considerar su aplicabilidad en el valle de Chaca.
\end{abstract}

Palabras clave: comparación de métodos, Hargreaves Samani 1985, Penman Monteith.

\begin{abstract}
Based on meteorological data obtained in the automatic weather station (AWS) placed in the "Chaca" sector, the Blaney Criddle method, radiation, Penman (1948), Hargreaves Samani (1985) and an evaporation tray with FAO-56 Penman Monteith method were compared in order to estimate the evapotranspiration (ET) in the Chaca valley located in the Arica and Parinacota region, Chile. The Blaney Criddle equation did not return satisfactory results (RMSE $6.35 \mathrm{~mm}$ per day and $R 2=67 \%$ ). The radiation method was the only one that underestimated the ET, because of the relative humidity in the area. There was a high R-squared (95\%) using the Penman equation (1948). Finally, the Hargreaves-Samani equation (1985) presented the best efficiency of estimation only using the air temperature and solar radiation, which allows considering its application in the Chaca valley.

Key words: evapotranspiration, Penman Monteith, Automatic Weather Station.
\end{abstract}

\section{Introducción}

En los valles de la Región de Arica y Parinacota se cultiva bajo condiciones de escasez hídrica (Trevizán, 2011), por otra parte, la alta radiación y vientos moderados a fuertes generan una alta demanda atmosférica, la que es fundamental cuantificar apropiadamente, en la medida de lo posible, de una forma simple y que permita resultados consistentes.

La evapotranspiración es un componente fundamental del balance hidrológico y un factor clave en la interacción de la superficie terrestre y la atmósfera. Su cuantificación se hace necesaria en contextos tan diferentes como la producción vegetal, la planificación y la gestión de recursos o estudios ambientales y ecológicos; depende, entre otros, de dos factores muy variables y difíciles de medir: el contenido de humedad del suelo y el desarrollo vegetal de la planta, por esta razón se introdujo el término evapotranspiración de referencia que se refiere a la tasa evapotranspiratoria de un cultivo de referencia (festuca o alfalfa), de altura uniforme y de activo crecimiento que no tiene restricciones de agua y cubre completamente el suelo, permaneciendo en óptimas condiciones de humedad (Allen et al., 2006).

Existen variados métodos para estimar directa o indirectamente la evapotranspiración de referencia. Los métodos directos se basan en la entrada y salida de agua en un cultivo específico, donde se obtiene la evapotranspiración real o similar, entre ellos se

\footnotetext{
1 Depto. Recursos Ambientales, Facultad de Ciencias Agronómicas, Universidad de Tarapacá.

* Autor por correspondencia: atorres@uta.cl
} 
mencionan los lisímetros, microlisímetros, balance de energía de Bowen y sistema de flujos turbulentos (Balbontin C. et al., 2011). Por otro lado, entre los métodos indirectos se denota a Penman Monteith FAO 56 que se utiliza para determinar los requerimientos hídricos de los cultivos, cuando se dispone de sistemas de registros automatizados (Maldonado y Aravena, 2006), además posee una precisión adecuada para diferentes zonas del mundo (Jensen et al., 1990). El objetivo del trabajo fue seleccionar un método indirecto alternativo al método de Penman Monteith FAO 56 para estimar en forma diaria la evapotranspiración de referencia en el valle de Chaca.

\section{Materiales y Métodos}

El trabajo se realizó durante agosto, septiembre y octubre de 2010 y se obtuvieron 88 datos observados $(\mathrm{N}=88)$. Se instaló una EMA (estación meteorológica automática) (DAVIS, Vantaje pro2) a 330 m.s.n.m el valle de Chaca, específicamente en el sector "Chaca", ubicada a $45 \mathrm{~km}$ de la ciudad de Arica (Chile), en las coordenadas $18^{\circ} 48^{\prime} \mathrm{S}-70^{\circ}$ $07^{\prime} \mathrm{O}$, donde se concentra la mayor parte de los agricultores del valle. Los datos obtenidos fueron procesados en planillas de cálculos en el software Microsoft Office Excel 2007. Para estimar la evapotranspiración de referencia en el valle de Chaca, se utilizaron métodos descritos en la Tabla 1.

La Figura 1 muestra que la bandeja de evaporación se instaló dentro de un cerco de protección junto a la EMA.

Los datos de la bandeja de evaporación fueron obtenidos en forma diaria (8:30 am) para hacer las comparaciones. Se calibraron dos recipientes de medida, uno de 1 litro y otro de $100 \mathrm{cc}$. Por otro lado la EMA se programó a registro horario. Los requerimientos de cada método son presentados en

Tabla 1. Métodos utilizados para estimar la evapotranspiración de referencia.

\begin{tabular}{|c|c|c|}
\hline Método & Fórmula & Coeficientes \\
\hline $\begin{array}{l}\text { Blaney Criddle FAO } 24 \\
\text { (Dorenboos y Pruit, 1977) } \\
\text { (Jensen } \text { et al., 1990) }\end{array}$ & Eto $=a+b * f$ & $\begin{array}{l}\mathrm{a}=0,0043 *(\operatorname{Rhmin}) *(\mathrm{n} / \mathrm{N})-1,41 \\
\mathrm{~b}=0,82 * 0,0041 *(\operatorname{Rhmin})+1,07 * \\
(\mathrm{n} / \mathrm{N}) * 0,066+\mathrm{u}_{2}-0,006 * \mathrm{RH} \min * \\
(\mathrm{n} / \mathrm{N})-0,0006 *(\mathrm{RHmin}) * \mathrm{u}_{2} \\
\mathrm{f}=\mathrm{p}(0,46 * \mathrm{~T}+8,13)\end{array}$ \\
\hline $\begin{array}{l}\text { Radiación FAO } 24 \\
\text { (Doorenbos y Pruit, 1977) } \\
\text { (Jensen } \text { et al., 1990) }\end{array}$ & $E t o=a+b *(\Delta / \Delta+\gamma) *(R s /)$ & $\begin{array}{l}\mathrm{a}=-0,3 \\
\mathrm{~b}=1,066-0,13 * 10^{-2} * \mathrm{Rhm}+0,045 \\
* \mathrm{u}_{2}-0,2 * 10^{-3} * \mathrm{Rhm} * \mathrm{u}_{2}-0,315^{*} 10^{-4} \\
* \mathrm{Rhm}^{2}-0,11 * 10^{-2} *\left(\mathrm{u}_{2}\right)^{2}\end{array}$ \\
\hline
\end{tabular}

\begin{tabular}{lcc}
\hline $\begin{array}{l}\text { Penman 1948 } \\
\text { (Penman H., 1948) }\end{array}$ & Eto $=\frac{\Delta *(R n-G)+\gamma * 6,43 * \int(u) *(e a-e d)}{\Delta+\gamma}$ & $\mathrm{f}(\mathrm{u})=0,27 *\left(1+\mathrm{U}_{2} / 100\right)$ \\
\hline Hargreaves-Samani 1985 & Eto $=0,0023 * R a *(T+17,89) * T D^{0,5}$ & $\mathrm{TD}=\left(\mathrm{T}^{\mathbf{o}}\right.$ max. $\left.\mathrm{T}^{\mathbf{o}} \min .\right)\left({ }^{\circ} \mathrm{C}\right)$.
\end{tabular}

(Allen et al., 2006)

Bandeja de Evaporación

(Allen et al., 2006)

$$
\text { Eto }=\text { Epan } * K p
$$

$\mathrm{Kp}=0,61+0,00341 * \mathrm{Rhm}-$

$0,000162 * \mathrm{u}_{2} * \mathrm{Rhm}-0,00000959 *$

$\mathrm{u}_{2} *$ BORDE $+0,00327 * \mathrm{u}_{2} * \ln (\mathrm{BORDE})$

$-0,00289 * \mathrm{u}_{2} * \ln \left(86,4 * \mathrm{u}_{2}\right) * \ln (\mathrm{BORDE})$

$+0,00063 *[\ln (\text { BORDE })]^{2} * \ln \left(86,4 * \mathrm{u}_{2}\right)$

Penman Monteith FAO 56
(Allen et al., 2006) $\quad$ Eto $=\frac{0,408 * \Delta *(R n-G)+\gamma * \frac{900}{T+273} * u_{2}(e s-e a)}{\Delta+\gamma *\left(1+0,34 * u_{2}\right)}$

Eto = Evapotranspiración sobre un cultivo de referencia $(\mathrm{mm} /$ día $) ; \mathrm{Rn}=$ radiación neta en la superficie del cultivo $\left(\mathrm{MJ} / \mathrm{m}^{2} /\right.$ día $) ;$ $\mathrm{G}=$ flujo de calor del suelo $\left(\mathrm{MJ} / \mathrm{m}^{2} /\right.$ día); $\mathrm{T}=$ temperatura promedio del aire a 2 metros de altura $\left({ }^{\circ} \mathrm{C}\right) ; \mathrm{u}_{2}=$ velocidad promedio diaria del viento a 2 metros de altura $(\mathrm{m} / \mathrm{s})$; es = presión de vapor en saturación $(\mathrm{kPa})$.

ea $=$ presión de vapor actual $(\mathrm{kPa}) ; \Delta=$ pendiente de la curva de presión de vapor versus temperatura $\left(\mathrm{kPa} /{ }^{\circ} \mathrm{C}\right)$.

$\gamma=$ constante psicrométrica $\left(\mathrm{kPa} /{ }^{\circ} \mathrm{C}\right) ; \mathrm{U} 2=$ Velocidad del viento $(\mathrm{km} /$ dia $) ; \mathrm{Ra}=$ Radiación extraterrestre $(\mathrm{mm} /$ día).

$\mathrm{T}^{\mathrm{o}} \max =\mathrm{T}^{\mathrm{o}}$ maxima $\left({ }^{\circ} \mathrm{C}\right) ; \mathrm{T}^{\circ} \min =\mathrm{T}^{\circ}$ minima $\left({ }^{\circ} \mathrm{C}\right) ; \mathrm{RHmin}=$ humedad relativa mínima $(\%) ; \mathrm{f}=$ Factor Blaney $-\mathrm{Criddle}(\mathrm{mm} /$ día); $\mathrm{p}$ = factor de horas de luz mensual; Rhm = Humedad relativa promedio $(\%)$; ea = Presión saturante del vapor de agua (milibares).; ed = Presión real del vapor de agua expresada (milibares); Epan = Evaporación de bandeja (mm/día); Kp = Coeficiente de Bandeja para suelo desnudo (adimensional); BORDE = Borde o distancia al borde de la superficie identificada (suelo en barbecho o cultivo seco en dirección del viento, caso B). 


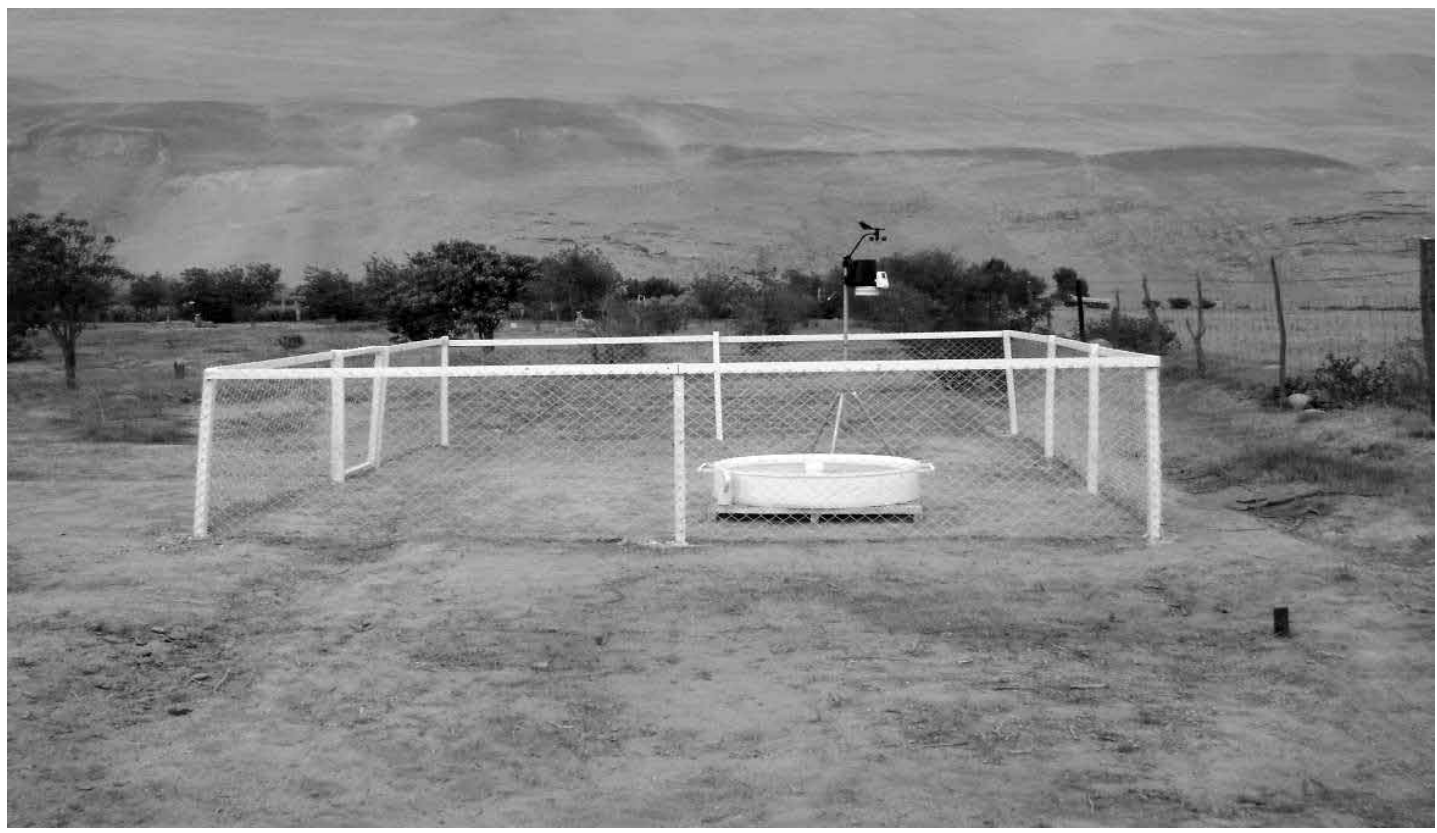

Figura 1. Estación agrometeorológica del valle de Chaca monitoreada bajo la supervisión de la Universidad de Tarapacá.

la Tabla 2, donde se observa que algunos métodos utilizan menos variables climáticas que otros.

El análisis se basó en la comparación de resultados obtenidos por los diferentes métodos, respecto de los obtenidos con Penman Monteith FAO 56, para ello se utilizó el coeficiente de correlación $(\mathrm{R})$ y el coeficiente de determinación $\left(\mathrm{R}^{2}\right)$. Para la determinación de la subestimación y sobreestimación se indicó el "Porcentaje de variación" con relación a Penman Monteith FAO 56.

$$
\% \text { Variación }=\left(\frac{E T o E m * 100}{E T o P M}\right)-100
$$

Donde:

$\%$ Variación $=$ Porcentaje de variación $(\%)$

$\mathrm{EToEm}=$ Evapotranspiración de referencia estimada con los métodos en estudio ( $\mathrm{mm} /$ día).

$\mathrm{EToPM}=$ Evapotranspiración de referencia media estimada con el método Penman Monteith FAO 56 (mm/día).

Además, para precisar la desviación de los métodos estudiados respecto de ETo PM, se utilizó el Error Medio (MBE) y raíz cuadrada de error cuadrático medio (RMSE), en cuanto más cercano a 0 sea su valor, mejor es la concordancia.

Tabla 2. Requerimientos de variables climáticas para cada método estimativo de evapotranspiración de referencia.

\begin{tabular}{|c|c|c|c|c|c|c|c|}
\hline Métodos & $\mathrm{T}^{\mathrm{o}}$ & HR & $\mathrm{U}$ & Rs & Horas de sol & Presión atm. & Bandeja de evaporación \\
\hline EtoBC Blaney Criddle & - & - & - & $X$ & - & $\mathrm{X}$ & $\mathrm{X}$ \\
\hline EtoRd Radiación & $\bullet$ & $\bullet$ & $\bullet$ & $\bullet$ & $\mathrm{X}$ & $\bullet$ & $\mathrm{X}$ \\
\hline EtoPn Penman 1948 & $\bullet$ & $\bullet$ & • & $\bullet$ & $\mathrm{X}$ & • & $\mathrm{X}$ \\
\hline EtoHG Hargreaves-Samani 1985 & • & $\mathrm{X}$ & $\mathrm{X}$ & $\bullet$ & $\mathrm{X}$ & $\mathrm{X}$ & $\mathrm{X}$ \\
\hline Etopan Bandeja de Evaporación & $\mathrm{X}$ & • & $\bullet$ & $\mathrm{X}$ & $\mathrm{X}$ & $\mathrm{X}$ & - \\
\hline EtoPM Penman Monteith FAO 56 & - & • & • & $\bullet$ & - & $\bullet$ & $\mathrm{X}$ \\
\hline
\end{tabular}

Fuente: Elaboración propia.

•: Requerido.

$\mathrm{X}$ : No requerido. 


$$
M B E=\frac{\sum \frac{N}{i=1}(E T O P M i-E T O E i)}{N}
$$

Donde:

MBE $=$ Error Medio (mm/día).

$\mathrm{ETPM}_{\mathrm{i}}=$ Evapotranspiración de referencia estimada con el método Penman Monteith FAO 56 (mm/día). ETOE $_{\mathrm{i}}=$ Evapotranspiración de referencia estimada con los métodos en estudio ( $\mathrm{mm} /$ día).

$$
R M S E=\sqrt{\frac{\sum \frac{N}{i=1}(E T P M i=E T O E i)^{2}}{N}}
$$

Donde:

RMSE = La raíz cuadrada del error cuadrático medio ( $\mathrm{mm} /$ día).

$\mathrm{ETPM}_{\mathrm{i}}=$ Evapotranspiración de referencia estimada con el método Penman Monteith. FAO 56 (mm/día). $\mathrm{ETOE}_{\mathrm{i}}=$ Evapotranspiración de referencia estimada con los métodos en estudio ( $\mathrm{mm} /$ día).

$\mathrm{N}=$ Número de observaciones.

\section{Resultados y Discusión}

Las estimaciones diarias de la evapotranspiración de referencia con los diferentes métodos de la FAO y Hargreaves-Samani 1985 sobrestiman la Eto bajo las condiciones del valle de Chaca, exceptuando el método Radiación donde subestimó por un 19,7\% la EtoPM. La Tabla 3 muestra los métodos evaluados frente al método de EtoPM FAO 56.

El método de Blaney Criddle tiende a sobrestimar en un 169,6\% gracias al progresivo aumento de las temperaturas y velocidades de viento de los meses de estudio (primavera-verano) aunque la humedad relativa mínima (Rhm) se presente entre 46 y $60 \%$, no fue determinante en la Eto. A pesar de que en la ecuación (EtoBC) se integraron más variables no se precisó la estimación de Eto. En cambio, Hargreaves Samani 1985 obtuvo la mínima sobrestimación respecto de los métodos de la FAO 24. El método de radiación fue el único que subestimó la ETo, se observó alta humedad relativa (HR) que se presentó en los meses de invierno y primavera (Tabla 4), ya que en verano comienza a disminuir sumado a una

Tabla 3. Evaluación de los métodos de estimación de ETo media diaria respecto del método de Penman Monteith FAO 56.

\begin{tabular}{lccccccrr}
\hline $\begin{array}{l}\text { Métodos } \\
\text { FAO }\end{array}$ & $\mathrm{N}$ & $\begin{array}{c}\text { Eto PM } \\
\text { Media }\end{array}$ & $\begin{array}{c}\text { Métodos FAO } \\
\text { Media }\end{array}$ & $\begin{array}{c}\text { Porcentaje } \\
\text { variación }\end{array}$ & $\begin{array}{c}\text { Correlación } \\
\text { simple }\end{array}$ & $\begin{array}{c}\text { Coeficiente } \\
\text { determinación }\end{array}$ & MBE & RMSE \\
\hline & & $\mathrm{mm} /$ día & $\mathrm{mm} /$ día & $\%$ & $\mathrm{R}$ & $\mathrm{R}^{2}$ & $\mathrm{~mm} /$ día & $\mathrm{mm} /$ día \\
\hline EtoBC & 88 & 3,4 & 9,2 & 169,6 & 0,82 & 0,67 & 5,79 & 6,35 \\
EtoRd & 88 & 3,3 & 2,6 & $-19,7$ & 0,98 & 0,95 & $-0,85$ & 0,88 \\
EtoPn & 88 & 3,3 & 4,5 & 37,8 & 0,97 & 0,94 & 0,18 & 1,10 \\
EToHG & 88 & 3,3 & 3,6 & 10,4 & 0,86 & 0,75 & 0,18 & 0,44 \\
Etopan & 88 & 3,3 & 4,5 & 37,7 & 0,89 & 0,79 & 1,07 & 1,36 \\
\hline
\end{tabular}

Fuente: Elaboración propia.

N: Número de observaciones; Blaney Criddle FAO 24 (EtoBC); Radiación FAO 24 (EtoRd); Penman 1948 (EtoPn); Hargreaves Samani 1985 (EtoHG), Bandeja de evaporación (Etopan) y Penman Monteith FAO 56 (EtoPM).

\begin{tabular}{|c|c|c|c|c|c|c|c|c|c|}
\hline Períodos & & $\begin{array}{l}\mathrm{T}^{\mathrm{o}} \\
\left({ }^{\circ} \mathrm{C}\right)\end{array}$ & & $\begin{array}{l}\mathrm{HR} \\
(\%)\end{array}$ & $\begin{array}{l}\text { Vel. viento } \\
(\mathrm{m} / \mathrm{s})\end{array}$ & $\begin{array}{l}\text { Radiación solar } \\
\text { (MJ/m²/día) }\end{array}$ & $\begin{array}{c}\text { Eto } \\
\text { (mm/día) }\end{array}$ & $\begin{array}{c}\text { Eto } \\
\text { (mm/día) }\end{array}$ & $\begin{array}{l}\text { Eto } \\
\mathrm{mm}\end{array}$ \\
\hline Mes & Media & Máx. & Mín. & Media & Media & Media & *PM & *PM mes & Acumulada \\
\hline Julio & 11,9 & 18,3 & 6,1 & 74 & 1,5 & 12,8 & 2,2 & - & - \\
\hline Agosto & 12,9 & 19,3 & 7,9 & 74,8 & 1,6 & 15,4 & 2,6 & 77,5 & 77,5 \\
\hline Septiembre & 14,8 & 19,7 & 10,1 & 72,3 & 2 & 14,6 & 2,8 & 90,2 & 167,7 \\
\hline Octubre & 15,8 & 22,1 & 9,8 & 69,5 & 2,2 & 23,5 & 4,1 & 127,1 & 294,9 \\
\hline Noviembre & 14,8 & 19,7 & 10,1 & 72,3 & 2 & 14,6 & 4,9 & 144,7 & 439,6 \\
\hline Diciembre & 20,8 & 28,5 & 13,1 & 59,8 & 2,3 & 24,9 & 5,7 & 168,8 & 608,4 \\
\hline
\end{tabular}

Tabla 4. Registro mensual de 2010 de las variables meteorológicas en el valle de Chaca, Arica-Chile.

Fuente: Elaboración propia. 
radiación solar (Rs) que aumenta gradualmente. Por el contrario, según (Maldonado y Selles, 2009) en los valles del interior en agosto, septiembre y octubre las HR son bien bajas en comparación con los valles intermedios y costeros, donde la Rs es media a alta, lo que hace suponer que determinar evapotranspiración de referencia con este método obtendríamos buenos resultados. Con Penman 1948 se obtuvo un $95 \%\left(\mathrm{R}^{2}\right)$ y una sobrestimación de $37,8 \%$ similar al obtenido con el método de la bandeja de evaporación, por lo cual se puede inferir que estos métodos por basarse en la evaporación de la lámina de agua libre tienden a sobrestimar la Eto. Finalmente, con Hargreaves Samani 1985 se obtuvo la mejor eficiencia de estimación respecto de Penman Monteith FAO 56 con 0,18 mm/día (MBE) y 0,44 mm/día (RMSE).

\section{Conclusiones}

- El método Hargreaves Samani 1985 obtuvo un mejor ajuste, permitiendo estimaciones más cercanas de la evapotranspiración de referencia en el valle de Chaca, con solamente dos variables, como la temperatura y radiación solar. Por lo tanto, se puede seleccionar esta fórmula para determinar "Eto" con bajo MBE y RMSE en el valle de Chaca.

- Los métodos como Blaney Criddle, Penman 1948 y radiación no son adecuados para la zona de estudio, ya que al calibrarse no presentan mejoría en la MBE y RMSE respecto de Hargreaves Samani 1985.

- La bandeja de evaporación sobrestima los valores de ETo.

\section{Bibliografía}

Allen, R.; Pereira, L.; Raes D. y Smith, M.

2006. Evapotranspiración del cultivo: Guías para la determinación de los requerimientos de agua de los cultivos. Cuadernos de riego y drenaje $\mathrm{N}^{\mathrm{0}} 56$. FAO. Roma. [citado 24 de mayo 2010], 298 p. Disponible en: <http://www.fao.org/docrep/009/ x0490s/x0490s00.htm>.

Balbontín, C.; Calera, A.; González, J.; Campos, I.; Llanos, M. y Torres, E.

2011, Comparación de los sistemas covarianza y relación de Bowen en la Evapotranspiración de un viñedo bajo clima semiárido, Agrociencia. Vol. 45: 87-103.

Doorenbos, J. y Pruitt, W.O.

1977.Guidelines for predicting crop water requirements, Estudio de Riego y Drenaje $\mathrm{N}^{\circ} 24$. Organización de las Naciones Unidas para la Agricultura y la Alimentación, Roma, 179 p. Jensen, M.; Burman, R. y Allen, R.

1990, Evapotranspiration and irrigation water requeriments ASCE-Manuals and Reports on Engineering practice, $\mathrm{N}^{\circ} 70$.
Maldonado, I. y Aravena, S.

2006, Redes de estaciones meteorológicas automáticas y sus aplicaciones productivas. Boletín INIA No 145 . Chile, $378 \mathrm{p}$.

Maldonado, I. y Selles, G.

2009, Instalación de redes de estaciones meteorológicas automáticas, para la Región de Arica y Parinacota, Instituto de Investigaciones Agropecuarias, Centro de Investigación Especializado en Agricultura del Desierto y Altiplano (CIE), INIA Ururi, Región de Arica y Parinacota. Ministerio de Agricultura.

Penman, H.C.

1948. Natural evapotranspiration from open water, bare soil and grass. Proc. R Soc. Lond. 193: 120-145 p.

Trevizán, J.

2011. Mirando sobre y bajo el agua. Idesia 29 (2): pp. 169174. 
